\title{
Parallel Appearance of Compulsive Behaviors and Artistic Creativity in Parkinson's Disease
}

\author{
Juho Joutsa $^{\mathrm{a}, \mathrm{b}} \quad$ Kirsti Martikainen $^{\mathrm{c}} \quad$ Valtteri Kaasinen $^{\mathrm{a}, \mathrm{b}}$ \\ ${ }^{a}$ Department of Neurology and ${ }^{\mathrm{b}}$ Turku PET Centre, University of Turku and \\ Turku University Hospital, and ${ }^{\mathrm{C}}$ The Finnish Parkinson Association and the \\ Finnish Parkinson Foundation, Turku, Finland
}

\section{Key Words}

Parkinson's disease - Impulse control disorder · Gambling · Punding · Mesolimbic . Dopamine $\cdot$ Ventral striatum $\cdot$ Creativity

\begin{abstract}
A 55-year-old male with idiopathic Parkinson's disease developed three behavioral changes under combination therapy with selegiline, cabergoline and levodopa. Co-existent behaviors included severe pathological gambling, punding and novel skills in writing poetry (published poetry books). Brain $\left[{ }^{18} \mathrm{~F}\right]$ fluorodopa PET imaging showed decreased tracer uptake in the striatum contralateral to the predominant motor symptoms, consistent with the clinical diagnosis of Parkinson's disease. Uptake in the ventral striatum was markedly high. Brain MRI before and after behavioral changes showed no pathological findings. The patient was diagnosed as having Parkinson's disease together with DSM-IV criteria-fulfilling pathological gambling and punding-like stereotyped behavior. There are no established criteria for the classification of emerged artistic creativity, although there are descriptions of the phenomenon in the literature. Inspired by the case, we conducted a preliminary survey including 290 patients with Parkinson's disease - exploring the possible relationship between creativity and impulsive-compulsive behaviors. The case, supported by the results of the survey, adds to the cumulative evidence of the association between dopaminergic medication and enhanced creativity, and suggests a possible linkage between increased artistic creativity and impulsive-compulsive behaviors in Parkinson's disease. Furthermore, it could be speculated that the high mesolimbic dopamine function might relate to the behavioral changes observed in this patient, and is suggestive of the overlapping neurobiological mechanisms of compulsive behaviors and artistic creativity.
\end{abstract}




\section{Introduction}

Over the past decade, it has become evident that Parkinson's disease and dopamine replacement therapy are associated with the increased frequency of impulse control disorders, such as pathological gambling, compulsive shopping, binge eating and hypersexuality [1]. Other repetitive behaviors, such as punding, walkabout and hobbyism, are also common in Parkinson's disease patients, and multiple behavioral problems occur frequently [2]. Anecdotal reports of changes in artistic creativity due to antiparkinsonian medication have been previously reported. For instance, a Parkinson's disease patient was reported to have developed novel poetic writing skills after starting drug treatment [3], and another patient started producing large amounts of artistic drawings [4]. Deep brain stimulation for Parkinson's disease has induced similar effects in one patient [5], and some patients, who had already shown artistic talent before the diagnosis, have experienced changes in artistic style either due to dopaminergic medication [6] or subthalamic stimulation [7]. It has also been suggested that a former artistic profession in Parkinson's disease patients could, in fact, be a risk factor for dopamine dysregulation syndrome, which is characterized by addiction to dopamine replacement therapy and stereotyped behaviors, such as punding [8].

\section{Case Report}

A 55-year-old right-handed man was diagnosed with Parkinson's disease in 2001 (onset at the age of 46 years). The first symptoms of the disease were rest tremor in the left hand and slight rigidity in the left upper extremity. Brain MRI at the time of the diagnosis showed no pathological findings. Antiparkinsonian medication was started at the time of the diagnosis. Medication changes over the course of the disease are presented in table 1. The patient had graduated from high school, corresponding to 12 years of formal education, and had various occupations after school including working as a janitor and a survey interviewer, before retiring due to motor symptoms of the disease 2 years after the diagnosis.

Approximately 5 years after the diagnosis, the patient gradually developed a gambling problem mostly for coin-operated slot machines. Before the diagnosis, the patient had no gambling problems and no experience in slot machine gambling. Earlier, while working as an interviewer in casinos, he remembered being 'surprised' that some people could get addicted to gambling. At the time of the brain PET imaging in 2010, he played more than $10 \mathrm{~h}$ /week on slot machines and spent more than $1,000 € /$ month on electronic machine gambling. His gambling had caused him significant debts. In 2010, pathological gambling was confirmed according to DSM-IV criteria. Maladaptive gambling behavior was indicated by 7 out of 10 DSM-IV items (cutoff $\geq 5$ items) and the South Oaks Gambling Screen (SOGS) score was 8 out of 20 (cutoff $\geq 5$ points). The questionnaire for impulsive-compulsive disorders in Parkinson's disease (QUIP) indicated pathological gambling ( 5 out of 5 items, cutoff $\geq 2$ items), but did not indicate any other impulse control disorders.

Together with slot machine gambling, the patient developed punding-like compulsory behavior, which involved picking wild berries. From the year 2006 onwards, he gradually spent more and more time picking berries. In 2010, the patient saw himself as being obsessed with picking wild berries together with the problematic gambling habit. In the autumn of 2010 alone, he picked hundreds of liters of lingonberries. He described going to the woods early in the morning, losing his sense of time and place (similar to gambling), and stayed in the woods for up to $12 \mathrm{~h}$. While picking, he felt no urge to gamble. He started picking berries before dawn, parking his car next to the woods and using the headlights of his car to find berries. In 2011, due to motor fluctuations and sudden off-periods, he had to regularly interrupt berry picking and return to his car for levodopa dosing.

The patient had shown no interest in arts or poetry before the diagnosis and dopamine replacement therapy, which was confirmed by his family and friends. Seven years after the diagnosis ( 2 years after gambling and punding started), he started writing poems. He predominantly wrote 
early in the morning (3-7 a.m.) before taking morning medications. The themes of his poems were a mixture of love, disease and nature. His poetry was considered interesting by a commercial publisher, and his first poetry book was published 1 year later, and the second book was published in 2010. He showed no interest in other forms of art, such as visual arts or music.

At the time of the PET imaging in 2010, the patient was on Hoehn and Yahr stage 2.0, and his total Unified Parkinson's Disease Rating Scale (UPDRS) score was 53 [UPDRS I = 2 (out of 16), UPDRS II = 12 (out of 52), UPDRS III = 36 (out of 108), UPDRS IV = 3 (out of 23)]. Blood count, plasma glucose, plasma alanine aminotransferase, plasma gamma-glutamyl transferase, fasting plasma creatinine and plasma carbohydrate-deficient transferring (CDT) were normal. Urine drug testing for cannabis, cocaine, opiates, phencyclidine, methadone, dextropropoxyphene and benzodiazepines was negative. Brain 1.5T MRI continued to show no pathological findings. The patient underwent a structured interview (SCID-I) by a consultant psychiatrist, which did not reveal any lifetime Axis-I psychiatric disorders or ADHD. The patient scored 10 points (out of 63) in Beck Depression Inventory, indicating that he did not suffer from clinically significant depression. Temperament and character inventory (TCI) scores were 27/40 (27 out of 40) for novelty seeking, 16/35 for harm avoidance, 20/24 for reward dependence, $5 / 8$ for persistence, $36 / 44$ for self-directedness, 34/42 for cooperativeness and $7 / 33$ for self-transcendence. Barratt Impulsiveness Scale (BIS-11) total score was 76, and subscores were 18 (range 8-32) for attentional, 23 (11-44) for motor and 35 (11-44) for non-planning impulsiveness.

Brain $\left[{ }^{18} \mathrm{~F}\right]$ fluorodopa-PET was performed with an ECAT EXACT HR+ scanner in 3D mode, injected dose $168 \mathrm{MBq}$, 90-min scanning, Patlak analysis with occipital cortex as reference, antiparkinsonian medications had been discontinued for $12 \mathrm{~h}$ prior to tracer injection, and carbidopa $150 \mathrm{mg}$ had been given orally $1 \mathrm{~h}$ prior to injection, as described previously [9]. Striatal deficit in tracer uptake was seen especially in the putamen contralateral to the predominant symptoms of the disease, which was in line with the diagnosis of idiopathic Parkinson's disease [right caudate $\mathrm{K}_{\mathrm{i}}\left(10^{-3} \mathrm{~min}^{-1}\right) 7.41$, left caudate 9.35, right putamen 2.99 , left putamen 4.21 , right ventral striatum 10.10 , left ventral striatum 10.40 , right medial orbitofrontal cortex (mOFC) 2.26, and left mOFC 2.36]. As compared to the other subjects of our previously reported study of Parkinson's disease patients without known increase in creativity, with or without impulse control disorders, the left [whole group $\mathrm{K}_{\mathrm{i}}$ median (range) 8.40 (6.46-10.40) $10^{-3} \mathrm{~min}^{-1}$ ] and right ventral striatal [8.77 (7.31-10.10)], and the left caudate [7.39 (4.87-9.35)], [18F]fluorodopa uptake of the present patient was the highest of the group $(\mathrm{n}=19$, one patient with self-reported increase in creativity was excluded from the analysis) [9].

After PET imaging, cabergoline was rapidly stopped due to pathological gambling. Soon after the stopping of cabergoline, pathological gambling behavior decreased without significant changes in punding or poetic creativity. In parallel with cabergoline discontinuation, his motor symptoms increased and restless legs symptoms appeared. The levodopa dose was therefore markedly increased in 2010 and low-dose pramipexole for restless legs syndrome was initiated (table 1). The patient felt that after cabergoline was stopped, his gambling was under control. Within 1 year of cabergoline discontinuation and levodopa dose increase in 2010, he developed significant motor fluctuations and is currently under consideration for deep brain stimulation.

Survey

Is the co-existence of the three behavioral changes coincidental in our patient? The possible linkage between impulsive-compulsive behaviors and artistic creativity in this patient led us to preliminarily investigate the phenomenon in a population of Parkinson's disease patients. We performed a postal survey inquiring the issue from January to August 2011. The survey was sent to 376 patients from our previous study [10] (patients who had indicated that they were willing to participate in further studies), which investigated the prevalence of impulse control disorders in Finnish patients with Parkinson's disease. The survey included the QUIP together with questions regarding possible artistic creativity before and after the diagnosis of Parkinson's disease. A total of $296(78.7 \%)$ of 376 patients returned the survey, but 6 patients were excluded because of inadequate data. Overall, $38.7 \%(n=108$ of 279$)$ of the patients screened positive for at least one impulse control disorder in QUIP and 19.3\% ( $n=54$ of 280) of the patients reported increased artistic creativity after the diagnosis of Parkinson's disease. Impulse control disorders as screened with QUIP were significantly more frequent in patients reporting increased creativity after the diagnosis than in patients without creativity increase (54.7 vs. $34.6 \%$, respectively; $n=270, \chi^{2}=7.31$, d.f. $=1, p=$ 0.007). Moreover, $33.3 \%$ (18 of the 54 ) of the patients with an increase in creativity subjectively 
linked the enhanced creativity directly to dopamine replacement therapy. The prevalence of creativity or impulse control disorders did not vary according to the side of predominant motor symptoms or to the type of medication used by the patient.

\section{Discussion}

The clinical symptoms and findings of the patient indicated the diagnosis of Parkinson's disease which was later supported by [18F]fluorodopa PET and normal findings in two brain MRIs, before and after the appearance of behavioral changes. The diagnosis of pathological gambling was confirmed according to DSM-IV criteria. To date, there are no generally accepted diagnostic criteria for punding, nor is it defined in the DSM-IV. However, the continuum of punding to hobbyism has been described as repetitive actions; from simple and meaningless behavioral rituals to those such as collecting, drawing, cleaning or categorizing, etc., overrunning physiological needs or social responsibilities, while patients may or may not enjoy the act or retain the insight of the irrationality of their behavior [11]. In this patient, the excessive berry picking clearly fulfilled the description above and was classified as punding, although the behavior was not completely stereotyped simple behavior without meaning. Furthermore, although the patient enjoyed berry picking, he subjectively felt that picking was compulsive.

In the context of Parkinson's disease, artistic productivity/creativity could be more related to the disease or the treatment than intrinsic artistic motivation. Although the increase in artistic creativity is multifactorial after the diagnosis of a chronic disease (including art produced in patient associations; the heightened interest in arts due to psychological factors after diagnosis and increased free time after premature retirement), the present data suggests that, irrespective of the cause, artistic creativity is associated with co-existent problems of impulse control in patients with Parkinson's disease. However, it should be noted that a previous study [12] provided contradictory results compared to ours, which could relate to differences in power to detect differences (number of patients 36 and 290, respectively), or in methodology as the previous study included only patients with completely novel artistic productivity, whereas the present study also included patients with prior artistic activity. It should also be noted that the survey was a retrospective study and that the positive screen for impulse control disorders with QUIP is not comparable to clinical diagnosis. We also lack clinical data to confirm self-reported Parkinson's disease diagnoses. Therefore, the results should be interpreted with caution and only as supportive material to the case described here.

Whether the increase in artistic production of our patient is a reflection of increased creativity, i.e. something that is novel and meaningful, or merely just an increase in basal arousal, can be questioned. Creativity has been defined as the ability to produce work that is at the same time novel and meaningful, as opposed to trivial or bizarre [13]. The poetry of the patient was accepted twice by a commercial publisher, which suggests that the artistic level of his work was above average. From a neurobiological and neuropharmacological perspective, one would expect dopamine-enhancing therapies to merely increase dopaminergic drive and activation. However, increased creative drive can secondarily improve creative skill [14]. First, there can be a practice effect: the more the patient paints, writes or acts, the better the quality of his work. 
Another option is that high motivation increases the number of ideas produced, and therefore, a proportional increase in the number of novel and exceptional ideas.

The patients showed markedly high novelty seeking and impulsiveness, as measured with TCI and BIS-11, compared to an earlier report including Parkinson's disease patients with and without impulse control disorders [15]. In addition, the PET imaging revealed exceptionally high ventral striatal and left caudate fluorodopa uptake suggesting high mesolimbic dopamine function, which has been speculated to be of importance in creativity [14] and impulse control disorders [11].

The patient had been on a maximal dose of cabergoline for 7 years and had suffered from pathological gambling and punding for 5 years before the treatment was stopped because of behavioral problems. As the result of the change in treatment, the urges for gambling decreased without changes in punding or creativity. There is literature to suggest that punding-like repetitive behaviors are particularly associated with levodopa treatment [2], whereas impulse control disorders, such as pathological gambling, are particularly associated with dopamine agonists [1]. Although one is tempted to interpret that, in this case, punding and creativity were related more to treatment with levodopa, and pathological gambling more to cabergoline, impulse control disorders and punding are known to frequently co-exist and there are multiple other factors, apart from medication, that are associated with repetitive behaviors in Parkinson's disease. If deep brain stimulation is performed for this patient in the future, a reduction in levodopa dose will be possible, and further behavioral changes could emerge.

The increased risk of impulse control disorders in Parkinson's disease seems to be associated not only with dopaminomimetic medication, but also with several other factors, such as younger age, smoking, family history of gambling problems, and depression $[1,10,15]$. Here we have described a patient with early-onset Parkinson's disease, who developed an impulse control disorder and punding together with novel poetic creativity. The case and our survey add to the cumulative evidence of the association between dopaminergic medication and enhanced creativity, and suggest a linkage between increased artistic creativity and impulsive-compulsive behaviors in Parkinson's disease. [18F]Fluorodopa PET imaging revealed markedly high mesolimbic dopamine function, which could relate to the behavioral changes described here, and is suggestive of the overlapping neurobiological mechanism of impulsive-compulsive behaviors and creativity. Our preliminary survey of 290 patients further suggests that the parallel appearance of these behaviors is not an exceptional rarity in Parkinson's disease. Larger epidemiological and neuroimaging studies are needed to investigate the relevance of these findings, with respect to the clinical characteristics and the neurobiological background of the phenomenon.

\section{Acknowledgements}

We are grateful to Hannele Hyppönen and the staff of Turku PET Centre for their skilled assistance during the study. This work was supported by the Finnish Alcohol Research Foundation, the Finnish Medical Foundation, the Turku University Central Hospital (EVO-funds), the Turku University Foundation, the Finnish Parkinson Foundation and the Paulo Foundation. 


\section{Disclosure Statement}

Dr. Joutsa has received speaker honoraria from Boehringer Ingelheim. Dr. Martikainen has received speaker honoraria from Boehringer Ingelheim, speaker honoraria and consultant fees from UCB Pharma, and consultant fees from Orion Pharma and GlaxoSmithKline. Dr. Kaasinen has received speaker honoraria and/or travel grants from Orion-Pharma, Abbott, UCB Pharma and Lundbeck; and serves as a member of the advisory board of UCB Pharma and as a consultant for Orion-Pharma and Lundbeck.

Table 1. Changes in the antiparkinsonian medication and the behavior of the patient

\begin{tabular}{|c|c|c|}
\hline $\begin{array}{l}\text { Time } \\
\text { after } \\
\text { diagnosis } \\
\text { years }\end{array}$ & Medication, daily dose & Non-motor behavioral changes \\
\hline 0 & Diagnosis of Parkinson's disease, selegiline 5 mg & - \\
\hline 1 & Selegiline $5 \mathrm{mg}$, cabergoline $1 \mathrm{mg}$ & - \\
\hline 2 & Selegiline $5 \mathrm{mg}$, cabergoline $2 \mathrm{mg}$ & - \\
\hline 3 & Selegiline $5 \mathrm{mg}$, cabergoline $2 \mathrm{mg}$, levodopa/carbidopa 100/25 mg & - \\
\hline 5 & Selegiline $10 \mathrm{mg}$, cabergoline $2 \mathrm{mg}$, levodopa/carbidopa 200/50 mg & Pathological gambling, punding \\
\hline 7 & Selegiline $10 \mathrm{mg}$, cabergoline $2 \mathrm{mg}$, levodopa/carbidopa 200/50 mg & $\begin{array}{l}\text { Creativity, pathological gambling, } \\
\text { punding }\end{array}$ \\
\hline 9 & $\begin{array}{l}\text { PET imaging, selegiline } 10 \mathrm{mg} \text {, cabergoline } 2 \mathrm{mg} \text {, levodopa/carbidopa } \\
300 / 75 \mathrm{mg}\end{array}$ & $\begin{array}{l}\text { Creativity, pathological gambling, } \\
\text { punding }\end{array}$ \\
\hline 10 & $\begin{array}{l}\text { Selegiline } 10 \mathrm{mg} \text {, pramipexole } 0.18 \mathrm{mg} \text {, levodopa/carbidopa/entacapone } \\
750 / 187.5 / 1,000 \mathrm{mg} \\
\text { under consideration for deep brain stimulation }\end{array}$ & Creativity, punding \\
\hline
\end{tabular}

\section{References}

1 Weintraub D, Koester J, Potenza MN, Siderowf AD, Stacy M, Voon V, Whetteckey J, Wunderlich GR, Lang AE: Impulse control disorders in Parkinson disease: a cross-sectional study of 3,090 patients. Arch Neurol 2010;67:589-595.

$\checkmark 2$ Spencer AH, Rickards H, Fasano A, Cavanna AE: The prevalence and clinical characteristics of punding in Parkinson's disease. Mov Disord 2011;26:578-586.

-3 Schrag A, Trimble M: Poetic talent unmasked by treatment of Parkinson's disease. Mov Disord 2001;16:1175-1176.

-4 Walker RH, Warwick R, Cercy SP: Augmentation of artistic productivity in Parkinson's disease. Mov Disord 2006;21:285-286.

5 Drago V, Foster PS, Okun MS, Haq I, Sudhyadhom A, Skidmore FM, Heilman KM: Artistic creativity and DBS: a case report. J Neurol Sci 2009;276:138-142.

6 Kulisevsky J, Pagonabarraga J, Martinez-Corral M: Changes in artistic style and behaviour in Parkinson's disease: dopamine and creativity. J Neurol 2009;256:816-819.

7 Witt K, Krack P, Deuschl G: Change in artistic expression related to subthalamic stimulation. J Neurol 2006;253:955-956.

-8 Schwingenschuh P, Katschnig P, Saurugg R, Ott E, Bhatia KP: Artistic profession: a potential risk factor for dopamine dysregulation syndrome in Parkinson's disease? Mov Disord 2010;25:493-496. 
9 Joutsa J, Martikainen K, Niemelä S, Forsback S, Rinne J, Kaasinen V: Increased medial orbitofrontal $\left[{ }^{18} \mathrm{~F}\right]$ fluorodopa uptake in Parkinsonian impulse control disorders. Movement Disorders 2012, in press, doi: $10.1002 / \mathrm{mds} .2941$.

10 Joutsa J, Martikainen K, Vahlberg T, Voon V, Kaasinen V: Impulse control disorders and depression in Finnish patients with Parkinson's disease. Parkinsonism Relat Disord 2012;18:155-160.

11 Evans AH, Strafella AP, Weintraub D, Stacy M: Impulsive and compulsive behaviors in Parkinson's disease. Mov Disord 2009;24:1561-1570.

12 Canesi M, Rusconi ML, Isaias IU, Pezzoli G: Artistic productivity and creative thinking in Parkinson's disease. Eur J Neurol 2012;19:468-472.

13 Sternberg RJ, Lubart TI: The concept of creativity: prospects and paradigms; in Sternberg RJ (ed): Handbook of Creativity. New York, Cambridge University Press, 2002.

$\checkmark 14$ Flaherty A: Frontotemporal and dopaminergic control of idea generation and creative drive. J Comp Neurol 2005;493:147-153.

15 Voon V, Sohr M, Lang AE, Potenza MN, Siderowf AD, Whetteckey J, Weintraub D, Wunderlich GR, Stacy M: Impulse control disorders in Parkinson disease: a multicenter case-control study. Ann Neurol 2011;69:986-996. 